have been tested and no systematic search for recombinants has been made. On this interpretation, $S p$ might turn out to belong either to $B$ or $F$ since Dodds and Long (1956) showed that $B I F$ were closely linked in that order. The $B$ and $F$ loci are both concerned with pigment distribution and it has been shown that there is a $B I$ position effect (Dodds, 1955).

\title{
3. Summary
}

1. The spectacle pattern is a white patch round each eye of an otherwise pigmented potato tuber. Genetically it is the heterozygote $I i^{s p}, I I$ being self-coloured and $i^{s p} i^{s p}$ white.

2. One self-coloured heterozygote has been detected and a rather consistent deficiency of spectacles in several families suggest that they may be fairly frequent (in the range 8-25 per cent. of heterozygotes).

3. Speculatively, it seems likely that $i^{s p}$ is compound.

\section{REFERENCES}

DoDds, K. s. 1955. Positional pseudo-alleles in diploid potatoes. Nature, 175, 394.

DODDs, K. S., AND LONG, D. H. 1956. The inheritance of colour in diploid potatoes II. 7. Genet., 54, 27-41.

DODDs, K. s., AND PAXMAN, G. J. 1962. The genetic system of cultivated diploid potatoes. Evolution, 16, 154-67.

HOWARD, H. W. 1967. Experiments on X-ray irradiation of potatoes. Abh. Deutsch. Akad. Wiss. Berlin, 1967, 311-7.

SIMmONDS, N. W. 1964. The genetics of seed and tuber dormancy in the cultivated potatoes. Heredity, 19, 489-504.

simmond, N. W. 1965. Somatic segregation of the spectacle pattern on potato tubers. Heredity, 20, 277-88.

\section{A CHROMOSOMAL CHIMERA IN A PLANT OF LILIUM CALLOSUM WITH A COMPLEX TRANSLOCATION*}

\author{
HIROSHI KAYANO \\ Department of Biology, Kyushu University;
}

Received 10.iii.69

\section{Introduction}

A chromosomal mutation in a somatic cell of a higher plant produces a cell-lineage of altered chromosomes, resulting in a chromosomal chimera (c.f. Swanson, 1957). Making use of chromosomal chimeras induced by X-ray, Brumfield (1943) determined the number of initial cells of the terminal meristem of roots to be three in Crepis capillaris and in Vicia faba. Davidson (1961) induced chromosomal chimeras in the meristem of regenerating roots of Vicia faba following X-irradiation and colchicine treatment, and estimated that the primordium of the root meristem was composed of 40-50 cells. In No. 171 .

* Contributions from the Department of Biology, Faculty of Science, Kyushu University, 
Lilium callosum Sieb. et Zucc. the present writer encountered a chromosomal chimera marked by cells with a complex translocation. This chimera is described below and evidence is presented to indicate that p.m.c.'s within a single anther are composed of multiple cell-lineages.

\section{Materials and methods}

A plant of $L$. callosum $(2 n=24)$ with a chromosomal chimera was found among 63 plants collected from a wild population growing near Karatsu, Saga Prefecture, Japan (population IV in Kayano, 1962). The chromosomes of root-tip cells and p.m.c.'s were prepared and observed using the same methods as described before (Kayano, 1956). This plant died before being used for further studies.

\section{Results AND DIsGUSSION}

Two kinds of p.m.c.'s were found in anthers from one flower, i.e. 1547 normal cells each with 12 II (plate I, fig. 1) and 73 aberrant cells each characterized by the presence of a small univalent (plate I, fig. 2). Of the latter type, 12 cells showed $1 \mathrm{~V}+9$ II +1 I (plate I, fig. 2; the pentavalent and the univalent in figs. $3 a, b$ ), three cells showed $1 \mathrm{III}+10 \mathrm{II}+1 \mathrm{I}$ (the trivalent and the univalent in figs. $4 a, b$ ), and in 58 cells the pairing configuration could not be analysed precisely, for some of the chromosomes were not separated well.
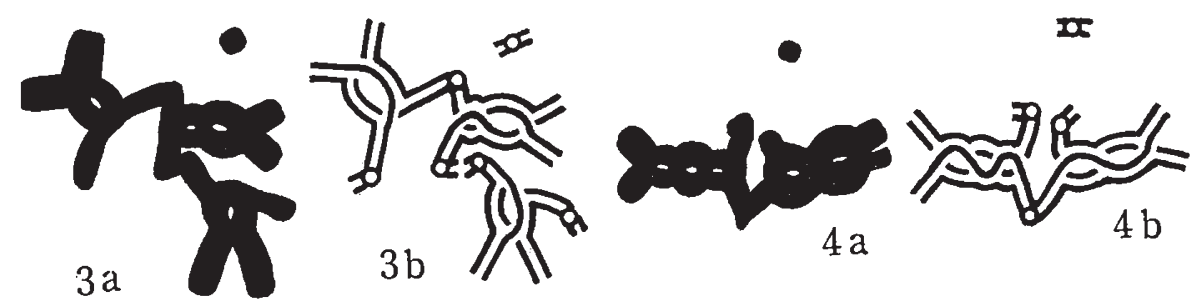

FIGS. $3 a$ and $b$.- The pairing configuration $1 \mathrm{~V}+1 \mathrm{I}$ in the p.m.c. shown in plate I, fig. 2 and the line diagram.

FIGs. $4 a$ and $b$. The pairing configuration 1 III +1 I from a p.m.c. showing 1 III +10 II +1 I and the line diagram.

The pairing configurations mentioned above suggests that the aberrant cells are heterozygous for a complex translocation involving segments of three non-homologous chromosomes (progressive or cyclic type of translocations; $c f$. fig. 29 in Burnham, 1962). That is, the three non-homologous chromosomes, $1 \cdot 2,3 \cdot 4$, and $5 \cdot 6$, each with a subterminal kinetochore (fig. $5 a$, have given rise to a large metacentric chromosome $(2 \cdot 4)$, a very small one $(5 \cdot 1)$ with a subterminal kinetochore, and one $(3 \cdot 6)$ morphologically indistinguishable from the unaltered condition (fig. $5 b$ ). A diagram of the possible pairing of the complex of six chromosomes at pachytene is shown in fig. 5c. At MI the complex of six chromosomes forms $1 \mathrm{~V}+1 \mathrm{I}$ (plate I, fig. 2; figs. $3 a, b$ ) without a chiasma in the arms 1 and 5 , or forms 1 III +1 II +1 I (cf. figs. $4 a, b$ ) without a chiasma in the arms 1,3 and 5 . 
Alternatively, the presence of maximum multiples of three and five chromosomes in different cells suggests that two independent reciprocal translocations have occurred, with one particular chromosome pair being

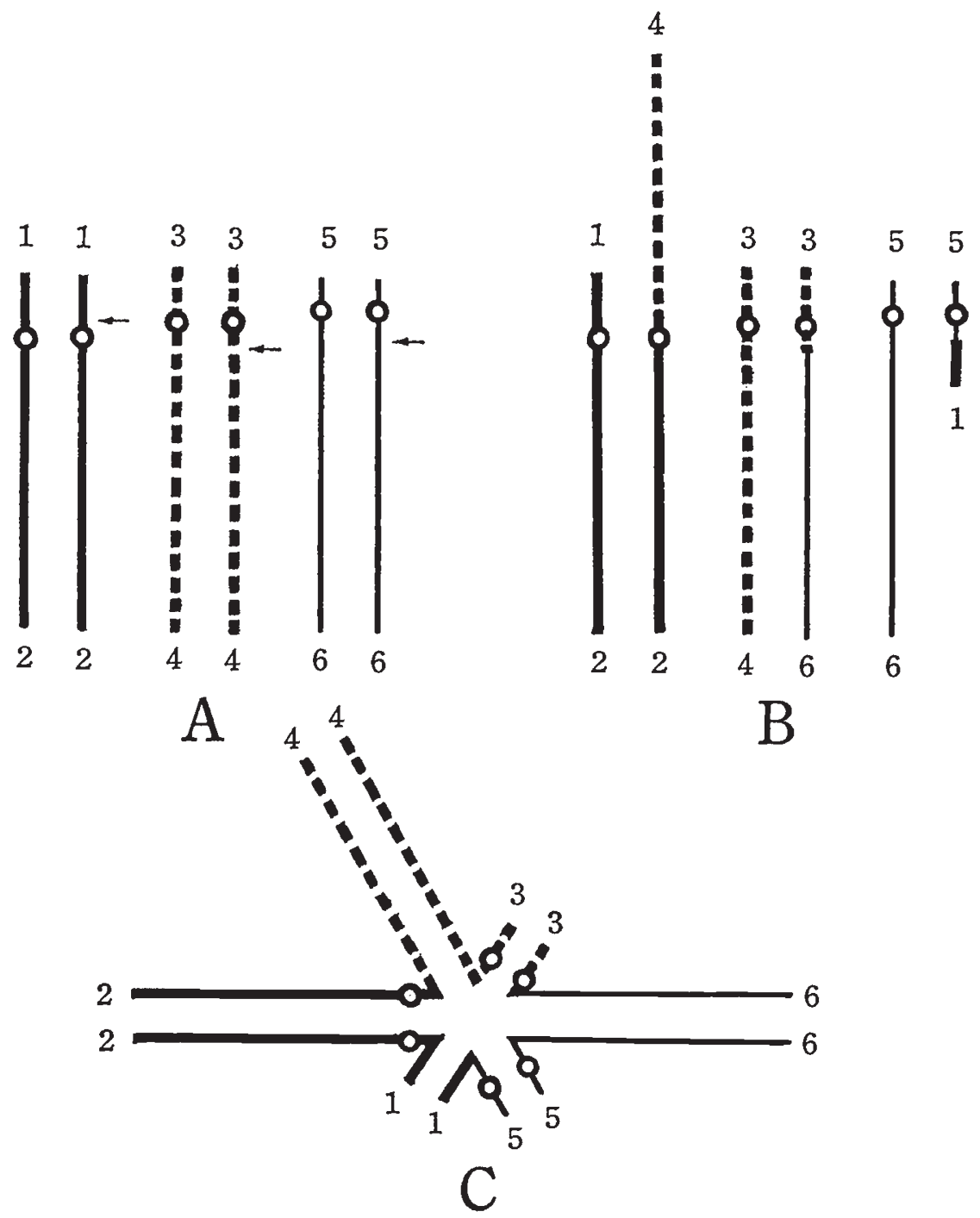

Frg. 5.-Diagrammatic representation of a complex translocation involving three pairs of chromosomes. (a) Three normal pairs in a somatic cell. Arrows indicate points of breakages. (b) A complex of six chromosomes established. (c) A possible pairing of the complex of six chromosomes at pachytene.

involved in both translocations. According to this view three kinds of celllineages would be present: 1 . Normal (12 II) ; 2. Heterozygous for one translocation (1 III +10 II $+1 \mathrm{I})$; 3. Heterozygous for both translocations $(1 \mathrm{~V}+9 \mathrm{II}+1 \mathrm{I})$.

Of the above two alternative explanations, the former is more likely to 
explain the present fact for the following reasons: 1 . The occurrence of the second translocation postulated in the latter explanation would occur in unaffected cell-lineages rather than would do in the cell-lineage affected by the first translocation. 2. If the second translocation had occurred in the same cell-lineage as the first translocation did, p.m.c.'s with maximum multiples of three chromosomes would be more frequent than those with maximum multiples of five chromosomes. This is not the case. The occurrence of $\odot 6$ (ring of six chromosomes) in p.m.c.'s in plants treated with X-ray, e.g. in barley (Caldecott and Smith, 1952) and in Tradescantia paludosa (Watanabe, 1959), and a configuration of chromatid exchanges involving three chromosomes in an X-irradiated embryonic cell of Schistocerca gregaria (fig. 52 in

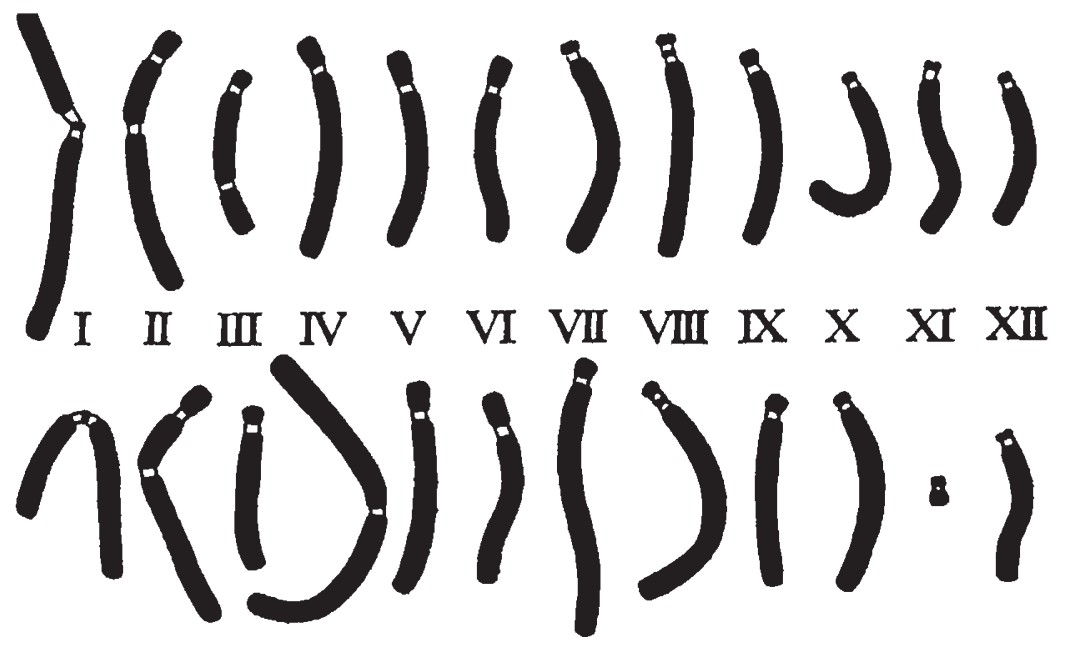

Fig. 6.-A complement of chromosomes from a root-tip cell of a chimerical plant showing normal and altered chromosomes.

Fox, 1967), are in favour of the former view. Furthermore, White (1963) has shown that a complex spontaneous translocation involving as many as four pairs of chromosomes, i.e. the occurrence of maximum multiples of eight chromosomes at meiosis, in a male of Moraba scurra, in which exchange of chromosomes occurred possibly in a gamete.

Three root-tip cells were analysed and the karyotype was found to be heterozygous for a translocation which corresponded exactly to that found in aberrant p.m.c.'s (fig. 6; cf. fig. 5b). This does not mean, however, that the plant was originally a translocation heterozygote and that the celllineage with normal chromosomes was produced by a complex translocation of chromosomes in a somatic cell because the complex of six chromosomes could hardly be reconstructed to the normal three pairs following breakages and reunions of the chromosomes. If more root-tips were available for analysis, the cells with normal complement might have been observed. Therefore, it is reasonable to conclude that this plant originally had normal cells and that a complex translocation occurred in a cell of the bulb which gave rise to a cell-lineage with the altered chromosomes in the shoot as well as in the root. 
In the present chimera, p.m.c.'s with normal chromosomes were 20 times as frequent as p.m.c.'s with altered chromosomes in one anther and 25 times as frequent in the other (table 1). This indicates that p.m.c.'s in a single anther are composed of multiple cell-lineages initiated by at least 20 cells.

TABLE 1

Frequencies of two kinds of p.m.c.'s in a plant of L. callosum

\begin{tabular}{cccr}
$\begin{array}{c}\text { Anther } \\
\text { no. }\end{array}$ & \multicolumn{2}{c}{ Type of p.m.c. } & $\begin{array}{r}\text { Total } \\
\text { cells }\end{array}$ \\
1 & 1077 & 55 & 1132 \\
2 & 470 & 18 & 488 \\
Total & 1547 & 73 & 1620
\end{tabular}

Reproduction of $L$. callosum is predominantly sexual, though it reproduces asexually at least in part ( $c f$. Kayano, 1962). The plant with the chromosomal chimera would produce chromosomal variation among the offspring through sexual reproduction, including the translocation heterozygotes and homozygotes which would be karyotypically distinct from the normal homozygotes. The occurrence of a complex translocation in the bulb suggests that a daughter bulb could produce plants heterozygous throughout for the complex translocation ( $c f$. Sharma, 1956). Watanabe (1959, 1962), for example, obtained various strains of complex translocation heterozygotes, i.e. those with $\odot 6+3 \mathrm{II}, \odot 8+2 \mathrm{II}, \odot 12$, etc. in p.m.c.'s following x-irradiation of axillary buds of the standard strain (6 II) of Tradescantia paludosa and by means of vegetative propagation.

\section{Summary}

1. A plant of Lilium callosum $(2 n=24)$ collected from a wild population was found to be a chromosomal chimera consisting of cells with normal chromosomes which formed 12 II in p.m.c.'s and those with altered chromosomes which formed $1 \mathrm{~V}+9 \mathrm{II}+1 \mathrm{I}$ or $1 \mathrm{III}+10 \mathrm{II}+1 \mathrm{I}$.

2 . The latter configurations and the karyotype of root-tip cells revealed that a complex translocation involving segments of three non-homologous chromosomes in a cell of the bulb gave rise to a cell-lineage with altered chromosomes in the shoot as well as in the root.

3. The relative frequency of two kinds of p.m.c.'s (normal and translocation) indicated that the p.m.c.'s in a single anther were composed of multiple cell-lineages initiated by at least 20 cells.

Acknowledgments. - The writer wishes to express his deep gratitude to Professor T. Haga, Kyushu University, for his suggestions and criticisms. The writer also gratefully acknowledges his indebtedness to Professor H. Lewis, University of California, Los Angeles, for kindly reading the manuscript and improving the sentences. Lastly, the writer is grateful to $\mathrm{Dr}$ G. H. Jones, University of Birmingham, for his comments on the manuscript.

\section{REFERENCES}

BRUMFIELD, R. T. 1943. Cell-lineage studies in root meristem by means of chromosome rearrangements induced by x-rays. Amer. 7. Bot., 30, 101-110.

burnham, c. R. 1962. Discussion in Cytogenetics. Burgess, Minneapolis. 
GALDECOTT, R. s., AND SMITH, L. 1952. A study of x-ray-induced chromosomal aberrations in barley. Cytologia, 17, 224-242.

DAvidson, D. 1961. Mechanism of reorganization and cell repopulation in meristem in root of Vicia faba following irradiation and colchicine. Chromosoma, 12, 484-504.

Fox, D. P. 1967. The effects of x-rays on the chromosomes of locust embryos. III. The chromatid aberration types. Chromosoma, 20, 386-412.

Kayano, H. 1956. Cytogenetic studies in Lilium callosum. I. Three types of supernumerary chromosomes. Mem. Fac. Sci. Kyushu Univ. Ser. E (Biol.), 2, 45-52.

xayano, H. 1962. Do. V. Supernumerary B chromosomes in wild populations. Evolution, $16,246-253$.

SHARMA, A. K. 1956. A new concept of a means of speciation in plants. Caryologia, 9, 93-130. swanson, c. P. 1957. Cytology and Cytogenetics. Prentice-Hall, Englewood Cliffs.

WATANABE, H. 1959. X-ray induced reciprocal translocations in Tradescantia paludosa. Japan. J. Genetics, 34, 162-167.

WATANABE, H. 1962. An X-ray-induced strain of ring-of-12 in Tradescantia paludosa. Nature, $193,603$.

WhITE, M. J. D. 1963. Cytogenetics of the grasshopper Moraba scurra. VIII. A complex spontaneous translocation. Chromosoma, 14, 140-145. 

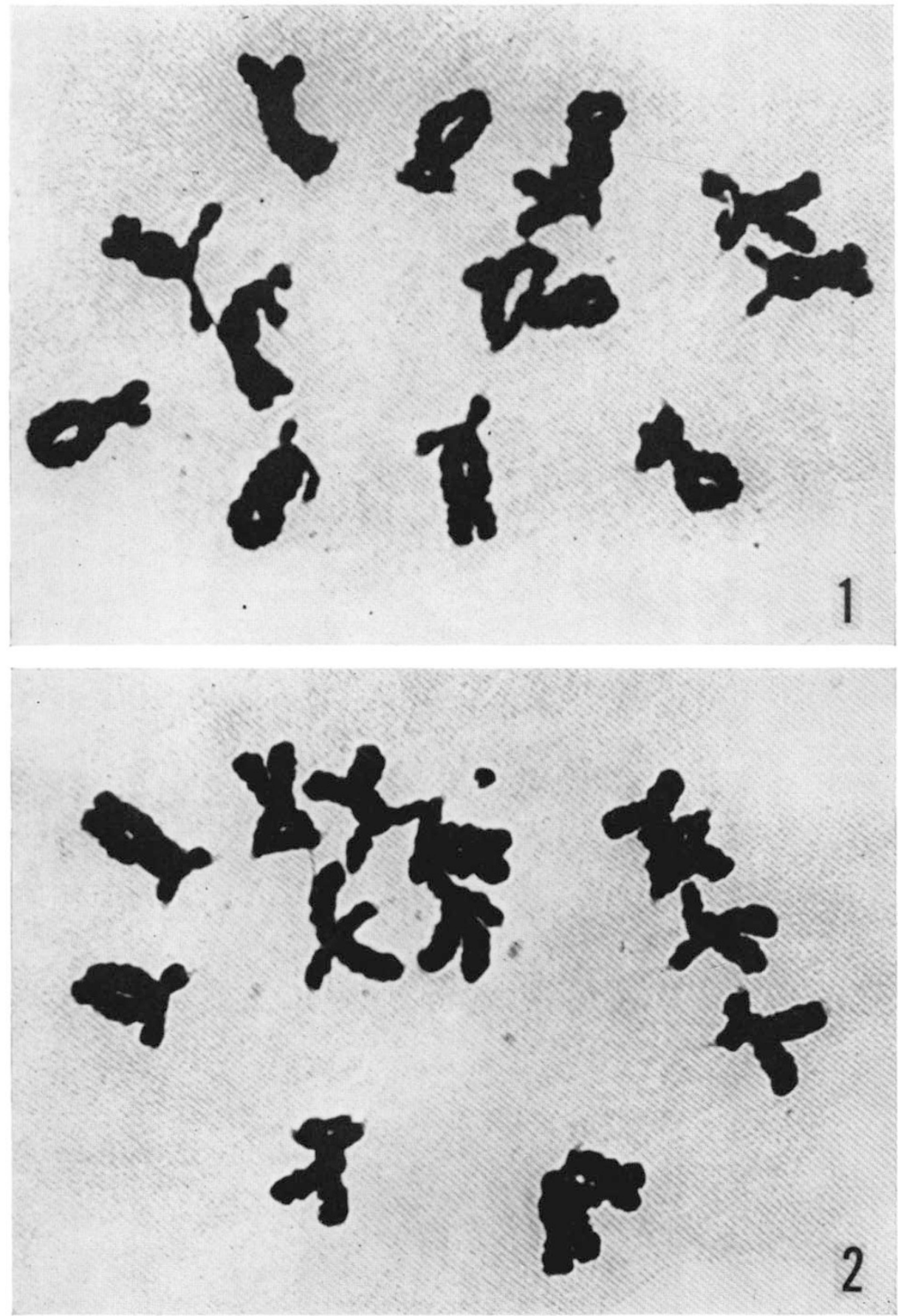

p.m.c.'s in a chromosomal chimera of Lilium callosum. Fig. 1, A normal p.m.c. showing 12 II. Fig. 2, An aberrant p.m.c. showing $1 \mathrm{~V}+9 \mathrm{II}+1 \mathrm{I} . \times 1400$. 